\section{Case Report}

Ann Liver Transplant 2021;1(1):100-104 https://doi.org/10.52604/alt.21.0015

Check for updates
ALT

pISSN 2765-5121

elSSN 2765-6098

\title{
Treatment of steroid-resistant acute rejection after living donor liver transplantation
}

Tae Beom Lee, Hyo Jung Ko, Jae Ryong Shim, Byung Hyun Choi, Kwangho Yang, Je Ho Ryu

Division of Hepato-Biliary-Pancreatic Surgery and Transplantation, Department of Surgery, Pusan National University Yangsan Hospital, Yangsan, Korea

Liver transplantation (LT) is the definitive treatment for end-stage liver disease. Acute rejection used to be a common complication up to $70 \%$ of recipients within the first year. Steroid pulse therapy is a helpful treatment for this complication but is not a preferred treatment for steroid-resistant acute rejection (SRAR). We report the successful treatment of patients diagnosed with steroid-resistant acute rejection. The patient, a 42-year-old male, diagnosed with chronic hepatitis $b$ related liver cirrhosis, underwent living donor liver transplantation on 28th December 2015. This patient was given $20 \mathrm{mg}$ basiliximab as induction therapy on days 0 and 4 post-transplantation. The immunosuppressive maintenance regimens for this patient included a double regimen (tacrolimus and steroid). At 20 months after transplantation, he was admitted to our hospital, presenting elevated serum levels of liver enzymes and total bilirubin. We performed the liver biopsy after vascular or biliary complications were excluded by computed tomography. A liver biopsy showed acute cellular rejection. Steroid pulse therapy was not effective. The liver biopsy was repeated to obtain an exact diagnosis. A second liver biopsy also confirmed acute cellular rejection. He was diagnosed with steroid-resistant acute rejection. He received $1.5 \mathrm{mg} / \mathrm{kg} /$ day anti-thymocyte globulin for 5 days. He received antihistamine and antipyretic before anti-thymocyte globulin infusion to reduce or prevent adverse effects of anti-thymocyte globulin. The patient was stopped tacrolimus and $5 \mathrm{mg} /$ $\mathrm{kg} /$ day ganciclovir; ceftazidime prophylaxis was given during anti-thymocyte globulin therapy. After anti-thymocyte globulin treatment, His liver enzymes and total bilirubin were decreased. He was discharged 34 days later and almost normalized his liver enzymes and total bilirubin. We have shown that anti-thymocyte globulin is safe and effective for treating steroid-resistant acute rejection, preventing graft loss of chronic rejection.

Keywords: Steroid resistant; Graft rejection; Liver transplantation; Immunosuppressive agents; Anti-thymocyte globulin 


\section{INTRODUCTION}

Liver transplantation has become the standard treatment of choice for end-stage liver disease. Calcineurin inhibitors and steroids are the primary immunosuppressive therapy after liver transplant in many transplant centers worldwide [1]. Although calcineurin inhibitors have potent immunosuppressive effects, acute cellular rejections and chronic rejections can still be encountered in patients who are taking calcineurin inhibitors after liver transplant even after appropriate blood drug levels are achieved [1]. The typical management of acute cellular rejection involves the optimization of baseline immunosuppressive therapy and methylprednisolone pulse therapy. Twenty-eight percent to $35 \%$ of patients who have acute cellular rejections do not respond to high-dose steroid therapy and require further intervention $[2,3]$. We herein report a case of the successful treatment of a patient diagnosed with steroid-resistant acute rejection (SRAR).

\section{CASE PRESENTATION}

The patient, a 42-year-old male, who diagnosed chronic hepatitis $b$ related liver cirrhosis, underwent living donor liver transplantation on 28th December 2015. This patient was given $20 \mathrm{mg}$ basiliximab as induction therapy on days 0 and 4 post-transplantation. The high dose methylprednisolone (500 $\mathrm{mg}$ was administered during the operation. The maintenance immunosuppressive regimens for this patient included a double regimen (tacrolimus and steroid). The

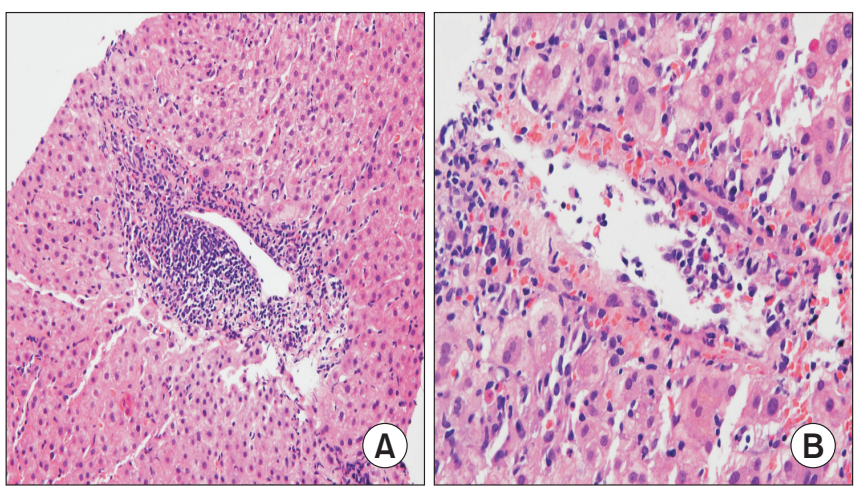

Fig. 1. (A) A biopsy before corticosteroid pulse therapy showed mild acute cellular rejection. The portal track was expanded because of a predominantly lymphocytes infiltration. (B) Central perivenulitis is characterized by an inflammatory infiltrate surrounding the central vein, which may or may not be associated with centrilobular hepatocyte injury, dropout, and necrosis. tacrolimus treatment was started at $6 \mathrm{hr}$ after LDLT, with a target of 8-12 $\mathrm{ng} / \mathrm{mL}$ during the first month after surgery and titration to $5-8 \mathrm{ng} / \mathrm{mL}$ over the next few months. After 1 year, a tacrolimus trough level was maintained at approximately $5 \mathrm{ng} / \mathrm{mL}$.

At 20 months after transplantation, he was admitted to our hospital presenting with the elevated serum levels of the liver enzymes (aspartate transaminase [AST] 604 IU/L and alanine transaminase [ALT] $1208 \mathrm{IU} / \mathrm{L}$ ) and total bilirubin $(8.9 \mathrm{mg} / \mathrm{dL})$. We performed the liver biopsy after vascular or biliary complications were excluded by computed tomography. The first liver biopsy showed acute cellular rejection (Fig. 1). This patient received steroid pulse therapy with methylprednisolone, $500 \mathrm{mg}$ per day for 3 days, and maintained with optimization of serum tacrolimus level between 8 to $10 \mathrm{ng} / \mathrm{mL}$. The serum level of the AST and AST was no improvement. We performed a repeated liver biopsy to obtain the exact diagnosis. The second liver biopsy also confirmed acute cellular rejection (Fig. 2). He was diagnosed with steroid-resistant acute rejection. He received $1.5 \mathrm{mg} / \mathrm{kg} /$ day anti-thymocyte globulin (ATG) for 5 days. He received antihistamine (chlorpheniramine $4 \mathrm{mg}$ ) and antipyretic (propacetamol $1 \mathrm{~g}$ ) before anti-thymocyte globulin infusion to reduce or prevent adverse effects of anti-thymocyte globulin. The patient was stopped tacrolimus and $5 \mathrm{mg} / \mathrm{kg} /$ day ganciclovir, ceftazidime prophylaxis was given during ATG therapy. After ATG, liver enzymes and total bilirubin were decreased. He was discharged 34 days later, and almost normalized liver enzymes and total bilirubin (Fig. 3).

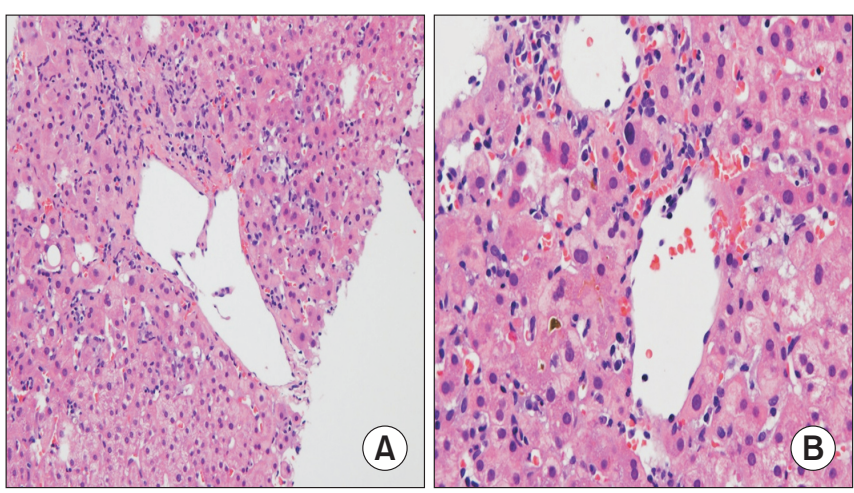

Fig. 2. (A) Biopsy after corticosteroid therapy showed remained perivenulitis. (B) Marked centrilobular intracytoplasmic and canalicular cholestasis, clinically evident with severe jaundice. 


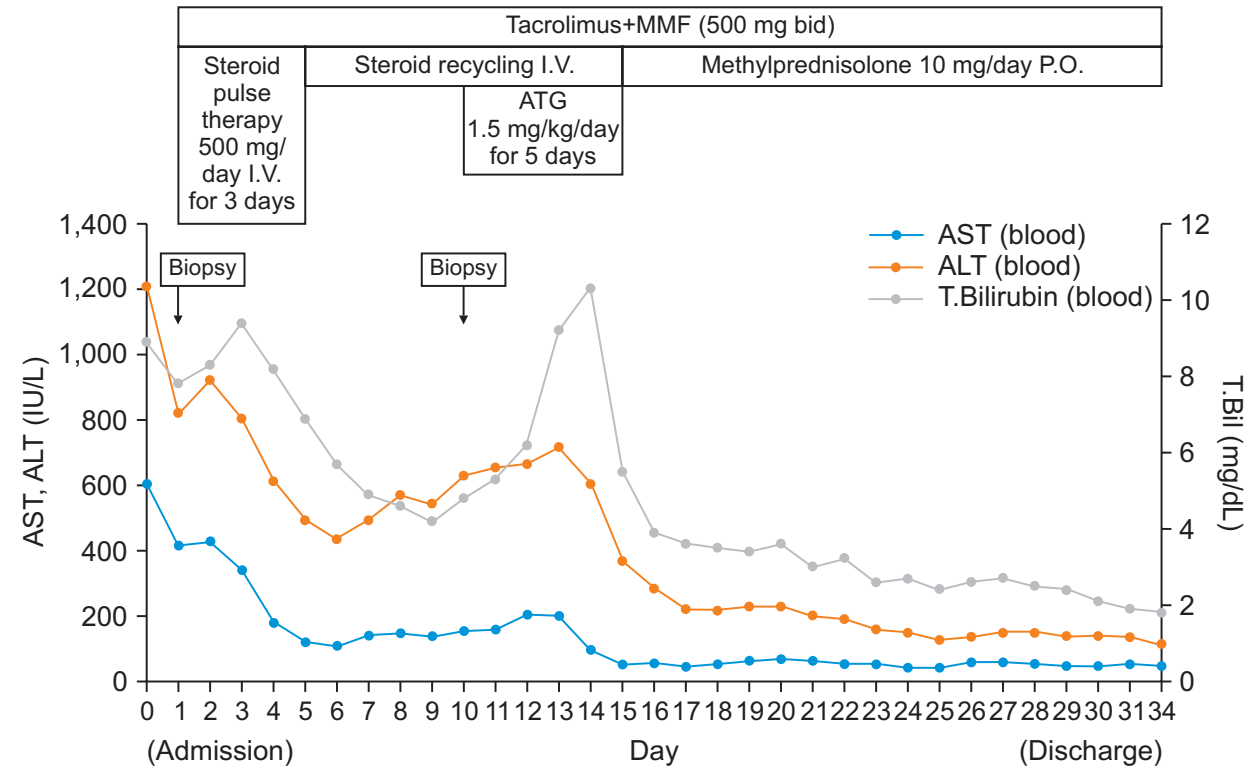

Fig. 3. Clinical course. MMF, mycophenolate mofetil; ATG, anti-thymocyte globulin; I.V., intravenous; P.O., per os; AST, aspartate transaminase; ALT, alanine transaminase; $T$. Bil, total bilirubin.

\section{DISCUSSION}

Because of the development of the liver transplant techniques, perioperative management, and application of new immunosuppressants, liver transplantation has been widely accepted as a definitive treatment for all kinds of end-stage liver disease [4]. Liver is an immune-privileged organ, and with the applying of current immunosuppressants, acute rejection after liver transplantation has been controlled to a low rate of $10-20 \%$. Moreover, more than $90 \%$ of rejection events could be invalidated by immunosuppressants and steroid bolus treatment $[5,6]$. Even though the incidence rate of SRAR is low, patients without appropriate treatment may suffer from graft loss or death. Various treatment strategies for SRAR have been provided by current literature.

Mycophenolate mofetil (MMF) has been used widely in liver transplantation to prevent acute rejection [7]. In a retrospective study of 191 patients with MMF administration, 38 of 47 patients with SRAR were normalized liver function tests by MMF treatment [8]. Aw et al. [9] showed that 21 of 28 pediatric transplant patients with SRAR also had positive responses to MMF treatment. MMF is well-known to cause gastrointestinal adverse effects in $24.6-52.5 \%$ of patients and bone marrow toxicity in $6.8-25.5 \%$ of patients. MMF also has a risk of infection and malignancy in a dose-dependent manner [8]. This use of an agent can be used only in selected patients and needs patient adaptation.

Interleukin-2 (IL-2) receptor antibody is mainly used to induce immunosuppressive therapy after liver transplantation to reduce the risk of acute rejection and renal damage due to early exposure to calcineurin inhibitors after liver transplantation [10]. IL-2 receptor antibodies have been reported as effective and well-tolerated in SRAR treatment. $[11,12]$ However, they need a larger sample to investigate as first-line therapy for the role of IL-2 receptor antibody for SRAR patients.

All of the cases mentioned above relied on traditional strategies for adequate immunosuppression. Nowadays, many institutions use triple immunosuppressant regimens, including tacrolimus, steroids, and, MMF and use induction immunosuppressants such as basiliximab. Hence, other more potent treatments, such as ATG and OKT3, are needed to treat SRAR.

Anti-T3-receptor antibody (OKT3) is a monoclonal antibody used as T-specific immunosuppressant to treat acute rejection of liver or kidney transplantation patients [13]. OKT3 has been reported to be helpful in the treatment of SRAR after kidney transplantation. Sevmis et al. [14] reported that OKT3 (5-10 mg/day, for 7 to 14 days) could effectively change $60-90 \%$ SRAR. Wu et al. [4] reported that 3 of 14 patients with SRAR after liver transplant showed hepatic liver function recovery after 2-3 days treatment, and SRAR was converted completely after treatment. However, OKT3 administration has side effects, such as cytokine release syndrome, infection, secondary malignancies, and the most severe complication is acute pulmonary edema [15]. Thus, the acute and severe side effects may be avoidable, while 
the long-term effects, especially infection, seem harder to avoid [4].

ATG, polyclonal antibody, is an effective induction agent and anti-rejection therapy in kidney transplantation [16]. Several studies with only small retrospective numbers of patients have reported effective results in the use of ATG for SRAR treatment in liver transplant recipients. Aydogan et al. [17] reported that 10 of 12 patients with SRAR were successfully treated using ATG therapy, and 2 patients advanced to chronic rejection and died after transplantation. 4 patients were experienced fever, chills which were the most common adverse effect during ATG treatment. However, they did not experience any serious reaction, serious infection, or long-term adverse outcomes after ATG treatment. Schmitt et al. [18] showed that 13 of 13 patients with SRAR were responded to ATG treatment. But, all hepatitis C virus (HCV) patients after administration of ATG developed recurrence with significant rises in HCV RNA levels. Two patients died from sepsis/multi-organ failure. Lee et al. [19] reported that 9 of 11 SRAR patients positive efficiency of ATG treatment. They reported one patient with HCV reactivation and one patient with fungemia. Three patients developed cytomegalovirus infections.

Although therapeutic effects of ATG on rescue therapy of SRAR are well known, they have significant adverse effects. Fever and chills were the most commonly encountered adverse effects during ATG treatment. The other adverse effects observed during ATG treatment were eruption, arthralgia, myalgia, headache, dizziness, abdominal pain, nausea, hypotension, leucopenia, and anemia. All these adverse effects were seen in the early period of ATG treatment, and they were managed by close monitoring of the patients, appropriate interventions like fluid administration, antihistaminics, antipyretics, nonsteroidal anti-inflammatory drugs, and prophylaxis. Anemia was treated with packages of red blood cells and leucopenia with GMCSF. There was no need to terminate treatment in any patient owing to adverse effects during ATG treatment. In the longer term, adverse effects of ATG, including opportunistic infections, development of malignancy, and recurrence of HCV can be encountered [17]. Our case-patient, he was not experienced early period adverse effects and long-term adverse effects.

In conclusion, ATG could be an excellent therapeutic option for liver transplantation recipients with SRAR. Indeed, a more extended follow-up period using ATG treatment is necessary to make a valid conclusion concerning the incidence of recurrent HCV, chronic and late-onset infections, and malignancies.

\section{FUNDING}

There was no funding related to this study.

\section{CONFLICT OF INTEREST}

All authors have no conflicts of interest to declare.

\section{ORCID}

Tae Beom Lee https://orcid.org/0000-0002-9603-965X Hyo Jung Ko https://orcid.org/0000-0003-0816-1725 Jae Ryong Shim https://orcid.org/0000-0002-9032-9373 Byung Hyun Choi https://orcid.org/0000-0002-9245-5066 Kwangho Yang https://orcid.org/0000-0003-1489-6787 Je Ho Ryu https://orcid.org/0000-0002-9377-3074

\section{AUTHORS' CONTRIBUTIONS}

Conceptualization: JHR. Data curation: HJK. Formal analysis: HJK. Funding acquisition: None. Investigation: None. Methodology: JRS. Project administration: BHC. Resources: JRS, BHC. Software: None. Supervision: JHR. Validation: KY. Visualization: KY. Writing - original draft: TBL. Writing - review \& editing: TBL.

\section{REFERENCES}

1. Busuttil RW, Lake JR. Role of tacrolimus in the evolution of liver transplantation. Transplantation 2004;77(9 Suppl):S44-S51.

2. Conti F, Dousset B, Archambeau D, Louvel A, Houssin D, Calmus $\mathrm{Y}$. Enhanced risk of steroid-resistant acute rejection following pretransplant steroid therapy in liver graft recipients. Transplantation 1995;60:1104-1108.

3. Andreu H, Rimola A, Bruguera M, Navasa M, Cirera I, Grande $\mathrm{L}$, et al. Acute cellular rejection in liver transplant recipients under cyclosporine immunosuppression: predictive factors of response to antirejection therapy. Transplantation 2002;73:1936-1943.

4. Wu L, Tam N, Deng R, Wu C, Chen P, Wang D, et al. Steroid-resistant acute rejection after cadaveric liver transplantation: experience from one single center. Clin Res Hepatol Gastroenterol 2014;38:592-597.

5. Neil DA, Hübscher SG. Current views on rejection pathology in liver transplantation. Transpl Int 2010;23:971-983.

6. Muñoz LE, Nañez H, Rositas F, Pérez E, Razo S, Cordero P, et al.; Liver Transplant Group. Long-term complications and survival of patients after orthotopic liver transplantation. Trans- 
plant Proc 2010;42:2381-2382.

7. Akamatsu N, Sugawara Y, Tamura S, Matsui Y, Kaneko J, Makuuchi M. Efficacy of mycofenolate mofetil for steroid-resistant acute rejection after living donor liver transplantation. World J Gastroenterol 2006;12:4870-4872.

8. Pfitzmann R, Klupp J, Langrehr JM, Uhl M, Neuhaus R, Settmacher $U$, et al. Mycophenolatemofetil for immunosuppression after liver transplantation: a follow-up study of 191 patients. Transplantation 2003;76:130-136.

9. Aw MM, Verma A, Rela M, Heaton N, Mieli-Vergani G, Dhawan A. Long-term outcome of mycophenolate mofetil rescue therapy for resistant acute allograft rejection in pediatric liver transplant recipients. Liver Transpl 2008;14:1303-1308.

10. Goralczyk AD, Hauke N, Bari N, Tsui TY, Lorf T, Obed A. Interleukin 2 receptor antagonists for liver transplant recipients: a systematic review and meta-analysis of controlled studies. Hepatology 2011;54:541-554.

11. Aw MM, Taylor RM, Verma A, Parke A, Baker AJ, Hadzic D, et al. Basiliximab (Simulect) for the treatment of steroid-resistant rejection in pediatric liver transpland recipients: a preliminary experience. Transplantation 2003;75:796-799.

12. Shigeta T, Sakamoto S, Uchida H, Sasaki K, Hamano I, Kanazawa $\mathrm{H}$, et al. Basiliximab treatment for steroid-resistant rejection in pediatric patients following liver transplantation for acute liver failure. Pediatr Transplant 2014;18:860-867.

13. Brusa P, Casullo R, Dosio F, Cattel L, Beltramini S, Chiappetta
$\mathrm{R}$, et al. OKT3 monitoring in the treatment of steroid-resistant acute rejection of hepatotransplant recipients. Eur J Drug Metab Pharmacokinet 1998;23:301-306.

14. Sevmis S, Emiroglu R, Karakayali F, Yagmurdur MC, Dalgic A, Moray $G$, et al. OKT3 treatment for steroid-resistant acute rejection in kidney transplantation. Transplant Proc 2005;37: 3016-3018.

15. Go MR, Bumgardner GL. OKT3 (muromonab-CD3) associated hepatitis in a kidney transplant recipient. Transplantation 2002;73:1957-1959.

16. Mehrabi A, Mood ZhA, Sadeghi M, Schmied BM, Müller SA, Welsch $\mathrm{T}$, et al. Thymoglobulin and ischemia reperfusion injury in kidney and liver transplantation. Nephrol Dial Transplant 2007;22 Suppl 8:viii54-viii60.

17. Aydogan C, Sevmis S, Aktas S, Karakayali H, Demirhan B, Haberal M. Steroid-resistant acute rejections after liver transplant. Exp Clin Transplant 2010;8:172-177.

18. Schmitt TM, Phillips M, Sawyer RG, Northup P, Hagspiel KD, Pruett $T L$, et al. Anti-thymocyte globulin for the treatment of acute cellular rejection following liver transplantation. Dig Dis Sci 2010;55:3224-3234.

19. Lee JG, Lee J, Lee JJ, Song SH, Ju MK, Choi GH, et al. Efficacy of rabbit anti-thymocyte globulin for steroid-resistant acute rejection after liver transplantation. Medicine (Baltimore) 2016; 95:e3711. 